\title{
ROOTS OF CERTAIN CLASSES OF POLYNOMIALS
}

\section{LOUIS WEISNER}

It is well known ${ }^{1}$ that if the roots of the polynomials $\phi(z)$ and $F(z)$ are real, so are the roots of the polynomial $\phi(D) F(z)$, where $D=d / d z$. This result has been applied to certain types of entire functions and trigonometric integrals. ${ }^{2}$ The following example illustrates the method employed. If

$$
f(z)=\sum_{k=0}^{n} c_{k} z^{k}
$$

is a polynomial whose roots $\lambda_{1}, \cdots, \lambda_{n}$ lie on the unit circle, then the roots of the polynomials

$$
F_{p}(z)=c_{n} \prod_{k=1}^{n}\left[(1+z / p)^{p}-\lambda_{k}(1-z / p)^{p}\right], \quad p=1,2, \cdots,
$$

lie on the axis of pure imaginaries. Therefore, if the roots of the polynomial $\phi(z)$ lie on the axis of pure imaginaries, so do the roots of the polynomials $^{3} \phi(D) F_{p}(z), p=1,2, \cdots$. Now the sequence of polynomials $\left\{F_{p}(z)\right\}$ converges uniformly in every finite region to the function

$$
F(z)=e^{-n z} f\left(e^{2 z}\right)=\sum_{k=0}^{n} c_{k} e^{(2 k-n) z},
$$

and the sequence $\left\{\phi(D) F_{p}(z)\right\}$ converges likewise to

$$
\phi(D) F(z)=\sum_{k=0}^{n} c_{k} \phi(2 k-n) e^{(2 k-n) z} .
$$

The roots of $\phi(D) F(z)$ therefore lie on the axis of pure imaginaries. Removing the innocuous factor $e^{-n z}$, and replacing $e^{2 z}$ by $z$, the following theorem results: If the roots of $f(z)$ lie on the unit circle, and the roots of $\phi(z)$ lie on the axis of pure imaginaries, then the roots of the polynomial

Presented to the Society, September 5, 1941; received by the editors June 6, 1941.

${ }^{1}$ Ch. Hermite, Nouvelles Annales de Mathématiques, vol. 5 (1866), p. 479; Pólya and Szegö, Aufgaben und Lehrsätze aus der Analysis II, p. 47, Problem 62.

2 See G. Pólya, Über trigonometrische Integrale mit nur reellen Nullstellen, Journal für die reine und angewandte Mathematik, vol. 158 (1927), pp. 6-18.

${ }^{3}$ Replacing $z$ by $i z$ it follows from the above theorem of Hermite that if the roots of $\phi(z)$ and $F(z)$ lie on the axis of pure imaginaries, so do the roots of $\phi(D) F(z)$. 


$$
g(z)=\sum_{k=0}^{n} c_{k} \phi(2 k-n) z^{k}
$$

lie on the unit circle.

This theorem provides us with a large class of polynomials whose coefficients are explicitly given and whose roots lie on the unit circle. As the theorem itself concerns polynomials, while the proof is transcendental, an elementary proof is desirable. An elementary proof, with extensions, follows.

From the polynomials $f(z)$ and $\phi(z)$, where $f(z)$ has the explicit form (1), construct the polynomial $g(z)$ defined by (2). Setting

$$
\psi(z)=\phi(2 z-n),
$$

we have

$$
g(z)=\sum_{k=0}^{n} c_{k} \psi(k) z^{k}
$$

If $\alpha_{1}, \cdots, \alpha_{m}$ are the roots of $\psi(z)$, so that

$$
\psi(z)=a \prod_{k=1}^{m}\left(z-\alpha_{k}\right)
$$$$
a \neq 0,
$$

then

$$
g(z)=a \prod_{k=1}^{m}\left(z D-\alpha_{k}\right) \cdot f(z)=\psi(z D) f(z) .
$$

Now suppose that the roots of $\phi(z)$ lie on the axis of pure imaginaries and the roots of $f(z)$ in the ring $r_{1} \leqq|z| \leqq r_{2}$, where $0 \leqq r_{1} \leqq r_{2}$. From the relation between $\phi(z)$ and $\psi(z)$ it is evident that the roots of $\psi(z)$ lie on the line $\Re z=n / 2$. Let $\alpha$ be a root of $\psi(z)$ and $\zeta$ a root of the equation

$$
z f^{\prime}(z)-\alpha f(z)=0 .
$$

If $\zeta$ is also a root of $f^{\prime}(z), \zeta$ is a multiple root of $f(z)$ and hence lies in the ring $r_{1} \leqq|z| \leqq r_{2}$. In the contrary case the centroid of $f(z)$ relative to $\zeta$ is different from $\zeta$. This centroid is $^{4} \zeta_{1}=\zeta-n f(\zeta) / f^{\prime}(\zeta)=(1-n / a) \zeta$, by (4). From $\Re \alpha=n / 2$, we infer that $|1-n / \alpha|=1$; therefore $\left|\zeta_{1}\right|=|\zeta|$. If $\zeta$ is outside the ring $r_{1} \leqq|z| \leqq r_{2}$, the circle with center at the origin which passes through $\zeta$ and $\zeta_{1}$ fails to separate the roots of $f(z)$, in violation of a theorem of Laguerre. ${ }^{5}$ Therefore all the roots of (4)

${ }^{4}$ E. Laguerre, Oeuvres, vol. 1, p. 56.

${ }^{5}$ Laguerre, loc. cit., p. 57, p. 134; Pólya and Szegö, loc. cit., p. 57, Problem 106. 
lie in the ring $r_{1} \leqq|z| \leqq r_{2}$. Applying this result repeatedly it follows from (3) that the roots of $g(z)$ lie in the ring.

THEOREM 1. If the roots of $\phi(z)$ lie on the axis of pure imaginaries, and the roots of $f(z)$ in the ring $r_{1} \leqq|z| \leqq r_{2}$, then the roots of $g(z)$ lie in this ring.

COROLLARY 1. If the roots of $\phi(z)$ lie on the axis of pure imaginaries, and the roots of $f(z)$ on the circle $|z|=r$, then the roots of $g(z)$ lie on this circle.

COROLlaRy 2. If the roots of $\phi(z)$ lie on the axis of pure imaginaries, the roots of the polynomial

$$
\sum_{k=0}^{n} \phi(2 k-n) z^{k}
$$

lie on the unit circle.

To prove the first corollary, take $r_{1}=r_{2}=r$. To prove the second, take $f(z)=1+z+\cdots+z^{n}$.

We suppose now that the roots of $f(z)$ lie in the circular region $|z| \leqq r$, and that the roots of $\phi(z)$ lie in the half-plane $\Re z \leqq 0$. We reëxamine (4), retaining our previous notation. If $\alpha=0$, the roots of (4) lie in the region $|z| \leqq r$ by the Gauss-Lucas theorem; hence we need only consider the case $\alpha \neq 0$. We may also suppose that $\zeta$ is not a root of $f^{\prime}(z)$.

We now have $\Re \alpha \leqq n / 2$; hence $|1-n / \alpha| \geqq 1$, and $\left|\zeta_{1}\right| \geqq|\zeta|$. If $|\zeta|>r$, then $\left|\zeta_{1}\right|>r$, and a circle may be drawn through $\zeta$ and $\zeta_{1}$ which fails to separate the roots of $f(z)$. As this result contradicts Laguerre's theorem, the region $|z| \leqq r$ includes all the roots of (4) and consequently all the roots of $g(z)$.

THEOREM 2. If the roots of $\phi(z)$ lie in the half-plane $\Re z \leqq 0$, and the roots of $f(z)$ lie in the circular region $|z| \leqq r$, then the roots of $g(z)$ lie in this circular region.

Theorem 2 may be extended to the case in which $\phi(z)$ is the limit of a sequence of polynomials whose roots lie in the half-plane $\Re z \leqq 0$ and which converges uniformly in every finite region. ${ }^{6}$ Likewise Theorem 1 and its corollaries are valid if $\phi(z)$ is the limit of a se-

\footnotetext{
${ }^{6}$ Entire functions which are limits of such sequences of polynomials are characterized by Eduard Benz, Über lineare, verschiebungstreue Funktionaloperationen und die Nullstellen von ganzen Funktionen, Commentarii Mathematici Helvetici, vol. 7 (1935), p. 246.
} 
quence of polynomials whose roots lie on the axis of pure imaginaries and which converges uniformly in every finite region.

Hunter College

\section{GENERALIZED LAPLACE INTEGRALS}

R. P. BOAS, JR.

We consider the linear space $\mathfrak{S}(c)$ whose elements are functions $f(z)$ $[z=x+i y]$ which are analytic for $x>c$ and satisfy

$$
\int_{-\infty}^{\infty}|f(x+i y)|^{2} d y \leqq M, \quad x>c,
$$

where the finite number $M$ depends on the function in question. It is well known that an element $f(z)$ of $\mathfrak{S}(c)$ has boundary values $f(c+i y)$ almost everywhere on $x=c$, and that $\mathfrak{S}(c)$ is a Hilbert space if the norm of $f(z)$ is defined by

$$
\|f(z)\|^{2}=\int_{-\infty}^{\infty}|f(c+i y)|^{2} d y .
$$

Furthermore, it is known $[5$, p. 8] that if $f(z) \in \mathfrak{S}(c)$, then $f(z)$ is representable as a Laplace integral for $x>c$, in the sense that there is a unique function ${ }^{1} \phi(t)$ with $e^{-c t} \phi(t) \in L^{2}(0, \infty)$ such that

$$
\lim _{T \rightarrow \infty}\left\|f(z)-\int_{0}^{T} e^{-z t} \phi(t) d t\right\|=0 ;
$$

we shall express (2) by writing

$$
f(z)=\int_{0}^{\infty} e^{-z t} \phi(t) d t, \quad x>c .
$$

It is easily verified that the integral in (3) converges in the ordinary sense for $x>c$. A Laplace integral may be regarded as a generalized power series; the object of this note is to generalize the integral representation (3) by replacing $e^{-z t}$ by a kernel $g(z, t)$ which is in some sense "nearly" $e^{-z t}$, just as power series $\sum a_{n} z^{n}$ have been generalized ${ }^{2}$ by replacing the functions $z^{n}$ by functions $g_{n}(z)$.

Presented to the Society, September 5, 1941; received by the editors May 24, 1941.

${ }^{1}$ Unique, that is, up to sets of measure zero.

${ }^{2}$ For a bibliography of this problem, see [1]. 